\title{
On a variant of the Hardy inequality between weighted Orlicz spaces
}

\author{
by \\ AgnieszKa KatAmajska and \\ Katarzyna Pietruska-PaŁuba (Warszawa)
}

\begin{abstract}
Let $M$ be an $N$-function satisfying the $\Delta_{2}$-condition, and let $\omega, \varphi$ be two other functions, with $\omega \geq 0$. We study Hardy-type inequalities

$$
\int_{\mathbb{R}_{+}} M(\omega(x)|u(x)|) \exp (-\varphi(x)) d x \leq C \int_{\mathbb{R}_{+}} M\left(\left|u^{\prime}(x)\right|\right) \exp (-\varphi(x)) d x,
$$

where $u$ belongs to some set $\mathcal{R}$ of locally absolutely continuous functions containing $C_{0}^{\infty}\left(\mathbb{R}_{+}\right)$. We give sufficient conditions on the triple $(\omega, \varphi, M)$ for such inequalities to be valid for all $u$ from a given set $\mathcal{R}$. The set $\mathcal{R}$ may be smaller than the set of Hardy transforms. Bounds for constants are also given, yielding classical Hardy inequalities with best constants.
\end{abstract}

\section{INTRODUCTION}

General framework and classical approach. Hardy type inequalities have been the subject of intensive research, going back to Hardy, who in the early 1920 's $([25,26])$ obtained inequalities of the form

$$
\int_{\mathbb{R}_{+}}|u(t)|^{p} t^{\alpha-p} d t \leq C \int_{\mathbb{R}_{+}}\left|u^{\prime}(t)\right|^{p} t^{\alpha} d t
$$

This inequality was generalized to

$$
\left(\int_{\mathbb{R}_{+}}\left|\int_{0}^{t} f(\tau) d \tau\right|^{q} d \mu(t)\right)^{1 / q} \leq C\left(\int_{\mathbb{R}_{+}}|f(t)|^{p} d \nu(t)\right)^{1 / p}
$$

with two nonnegative Radon measures $\mu, \nu$, and further to inequalities in the Orlicz-space setting:

2000 Mathematics Subject Classification: Primary 26D10; Secondary 46E35. Key words and phrases: Hardy inequalities, weighted Orlicz spaces. 


$$
\begin{aligned}
& Q^{-1}\left(\int_{\mathbb{R}_{+}} Q(\omega(x)|T f(x)|) r(x)\right.d x) \\
& \leq P^{-1}\left(\int_{\mathbb{R}_{+}} P(C \varrho(x)|f(x)|) v(x) d x\right) .
\end{aligned}
$$

Inequalities in $L^{p}$ were studied by Muckenhoupt [46], Maz'ya and Rozin [44], Bradley [8], Kokilashvili [33], Sinnamon [53], Sawyer [51], Bloom and Kerman [6], Stepanov [54], and many others (we refer to the monographs $[38,36,37,34,45]$ and references therein). As to the Orlicz-space result (1.2), several authors contributed to a complete characterization of admissible weights $\omega, r, \varrho, v$ and nondecreasing functions $P, Q$ which allow for $(1.2)$ with $T f(x)=\int_{0}^{x} K(x, y) f(y) d y$ being the generalized Hardy operator with kernel $K$. To name just a few, we refer to the papers of Bloom and Kerman [5, 6], Lai [40, 41, 42, 43], Heinig and Maligranda [28], Heinig and Lai [27] and their references. For a more detailed account of such results, we refer to Sections 3.1 and 3.2 .

Hardy-type inequalities are widely applicable in PDE theory and functional analysis. For example, one can derive various Sobolev embedding theorems in the $L^{p}$ setting, which can then be used to prove the existence of solutions of the Cauchy problem for elliptic and parabolic PDEs (see e.g. $[9,22,36,15,44,55,39])$, to study the asymptotic behaviour of solutions $[2,57]$, as well as their stability $[10,11]$. They are present in probability theory (see e.g. $[17,50,19])$. Hardy inequalities are also interesting in their own right (see e.g. $[37,38,56]$ ). For latest results, see the very recent papers $[9,13,23,47]$ and their references.

Investigation of weighted or nonweighted Orlicz-Sobolev spaces defined by an $N$-function different from $\lambda^{p}$ is suggested by physical models (see e.g. $[1,3,14,48,15])$. Therefore it is worthwhile to examine Hardy-type inequalities in general Orlicz spaces as well.

One of the central problems around Hardy inequalities can be expressed as follows. Consider the sets $\mathcal{H}$ of Hardy transforms and $\mathcal{H}^{*}$ of conjugate Hardy transforms:

$$
\begin{aligned}
\mathcal{H} & =\left\{u(t)=\int_{0}^{t} f(s) d s: \int_{0}^{a}|f(\tau)| d \tau<\infty \text { for every } a>0\right\} \\
& =\left\{u \in W_{\mathrm{loc}}^{1,1}\left(\mathbb{R}_{+}\right): \lim _{r \rightarrow 0} u(r)=0\right\}, \\
\mathcal{H}^{*} & =\left\{u(t)=\int_{t}^{\infty} f(s) d s: \int_{a}^{\infty}|f(\tau)| d \tau<\infty \text { for every } a>0\right\} \\
& =\left\{u \in W_{\mathrm{loc}}^{1,1}\left(\mathbb{R}_{+}\right): \lim _{r \rightarrow \infty} u(r)=0\right\} .
\end{aligned}
$$

and let $T f$ be either the Hardy transform of $f$, or the conjugate Hardy transform of $f$. Then the following problem arises. 
Problem 1 (classical). Given two $N$-functions $Q, P$, describe all possible weights $(\omega, r, \varrho, v)$ for which inequality (1.2) holds with some constant $C$ independent of $f$ (with $T$ the Hardy transform or the conjugate Hardy transform).

This problem has been completely solved for the Hardy transform by Bloom and Kerman in [6] for modular functions $P$ and $Q$ such that $Q$ dominates $P$ in some special sense (for the details see Section 3.2). A further generalization of (3.3), without the domination restriction, can be found in Lai's paper [43]. Therefore Problem 1 can be considered as settled.

Another approach and its motivation. We are concerned with another problem, which is expressed as follows.

Problem 2 (general). Given two $N$-functions $Q, P$ and weights $(\omega, r, \varrho, v)$, find a possibly large set $\mathcal{R}$ contained in the set of locally absolutely continuous functions for which the inequality (1.2) holds with some constant $C$ independent of $u \in \mathcal{R}$.

Let us make some comments here.

It can happen that the given weights $(\omega, r, \varrho, v)$ obey the known requirements for the validity of (1.2), say for $T f$ being the Hardy transform of $f$, as described in Problem 1. In that case the set $\mathcal{R}$ contains the full set $\mathcal{H}$ of Hardy transforms. On the other hand, if this requirement is not satisfied, we cannot expect (1.2) to hold for every $u \in \mathcal{H}$. In such a case $\mathcal{R} \cap \mathcal{H}$ will be a proper subset of $\mathcal{H}$. The solution to Problem 2 would therefore lead to Hardy-type inequalities in a (possibly) narrower class of functions.

If we substitute $u=T f$ in (1.2), where $T$ is either the Hardy transform or the conjugate Hardy transform, then $u^{\prime}=f$, so that (1.2) reduces to the special case of (1.2).

The reduced problem and partial answers. In this paper we deal with a special variant of Problem 2, which reads as follows.

Problem 3 (reduced). Given an $N$-function $M$ satisfying the $\Delta_{2}$-condition, and a pair of functions $(\omega, \varphi)$ where $\omega \geq 0$, describe a possibly large set $\mathcal{R}$ contained in the set of locally absolutely continuous functions for which the inequality

$$
\int_{\mathbb{R}_{+}} M(\omega(x)|u(x)|) \exp (-\varphi(x)) d x \leq C \int_{\mathbb{R}_{+}} M\left(\left|u^{\prime}(x)\right|\right) \exp (-\varphi(x)) d x
$$

holds with some constant $C$ independent of $u \in \mathcal{R}$.

Variants of (1.4) with $M(\lambda)=\lambda^{p}$ and $\mathcal{R}$ determined by constraints concerning $M, \omega, \phi$ have been studied e.g. in [4, 16, 20,21] (see also their references). To the best of our knowledge their extension to the Orlicz space setting has not been considered so far. 
Our main result (Theorem 2.1) states that if the triple $(\omega, \varphi, M)$ satisfies certain simple compatibility conditions, then we can indicate a set $\mathcal{R}$ such that (1.4) holds for every $u \in \mathcal{R}$. Moreover, we give some bounds on the constant $C$, which can be expressed in terms of the Simonenko lower and upper indices (see [52] and [24], [18] for interesting related results).

The condition $u \in \mathcal{R}$ depends on the behaviour of $u$ near zero and near infinity, which is very natural in problems arising from PDEs: when analyzing a particular equation one can often say that its solution (i.e. our function $u$ ) has some "good" properties near the boundary, expressed in terms of its rate of decay near the boundary.

As an illustration we derive the classical Hardy inequalities (1.1) with best constants (see Section 3.1.1 for discussion).

We also obtain sufficient conditions for (1.4) to hold for every $u \in \mathcal{H}$ (see Proposition 5.2 in Section 5.2.1). These conditions can be easily implemented in practice, and since the verification of the classical Bloom-Kerman conditions (5.6) from [6] seems rather hard, by using our approach one can avoid that verification and quickly deduce that (1.4) is satisfied for every $u \in \mathcal{H}$.

Perhaps it is even more interesting to deal with the case when the BloomKerman conditions (5.6) are not satisfied, so that inequality (1.4) is not valid for all $u \in \mathcal{H}$. Then we find a set $\mathcal{R}$ such that $\mathcal{R} \cap \mathcal{H}$ is smaller than $\mathcal{H}$ and (1.4) holds for all its elements (see Section 5.2.2).

As a particular type of inequalities like (1.4), we analyze those with $\omega=\left|\varphi^{\prime}\right|$ (see Section 3.3), and in the class of admissible $\varphi$ 's we obtain the inequality

$$
\int_{\mathbb{R}_{+}} M\left(\left|\varphi^{\prime}(x)\right| u(x) \mid\right) \exp (-\varphi(x)) d x \leq C \int_{\mathbb{R}_{+}} M\left(\left|u^{\prime}(x)\right|\right) \exp (-\varphi(x)) d x .
$$

For $M(\lambda)=\lambda^{p}$ and $\psi(x)=\exp \{-\varphi(x) / p\}$ we get

$$
\int_{\mathbb{R}_{+}}\left(\left|\psi^{\prime}(x) u(x)\right|\right)^{p} d x \leq C \int_{\mathbb{R}_{+}}\left(\left|u^{\prime}(x) \psi(x)\right|\right)^{p} d x,
$$

which is nothing other than a particular case of the Caccioppoli inequality on $\mathbb{R}^{+}$(see e.g. $[12,29]$ ). Caccioppoli inequalities are commonly used in regularity theory, and so we believe that our variant (1.5) can be used in regularity theory as well.

\section{PRELIMINARIES AND STATEMENTS OF MAIN RESULTS}

\subsection{Preliminaries}

Orlicz spaces. Let us recall some preliminary facts about Orlicz spaces, referring e.g. to [49] for details. Here we deal with Orlicz spaces of functions 
defined on $\mathbb{R}_{+}$. Suppose that $\mu$ is a positive Radon measure on $\mathbb{R}_{+}$and let $M:[0, \infty) \rightarrow[0, \infty)$ be an $N$-function, i.e. a continuous convex function satisfying $\lim _{\lambda \rightarrow 0} M(\lambda) / \lambda=0$ and $\lim _{\lambda \rightarrow \infty} M(\lambda) / \lambda=\infty$.

The weighted Orlicz space $L_{\mu}^{M}$ we deal with is

$L_{\mu}^{M}:=\left\{f: \mathbb{R}_{+} \rightarrow \mathbb{R}\right.$ measurable :

$$
\left.\int_{\mathbb{R}_{+}} M(|f(x)| / K) d \mu(x) \leq 1 \text { for some } K>0\right\},
$$

equipped with the Luxemburg norm

$$
\|f\|_{L_{\mu}^{M}}=\inf \left\{K>0: \int_{\mathbb{R}_{+}} M(|f(x)| / K) d \mu(x) \leq 1\right\} .
$$

This norm is complete and turns $L_{\mu}^{M}$ into a Banach space. When $\mu$ is the Lebesgue measure, it is dropped from the notation. For $M(\lambda)=\lambda^{p}$ with $p>1$, the space $L_{\mu}^{M}$ coincides with the usual $L_{\mu}^{p}$ space (defined on $\mathbb{R}_{+}$).

The symbol $M^{*}$ denotes the complementary function of an $N$-function $M$, i.e. its Legendre transform: $M^{*}(y)=\sup _{x>0}[x y-M(x)]$ for $y \geq 0$. It is again an $N$-function and from its definition we get the Young inequality

$$
x y \leq M(x)+M^{*}(y) \quad \text { for } x, y \geq 0 .
$$

$M$ is said to satisfy the $\Delta_{2}$-condition if, for some constant $c>0$ and every $\lambda>0$, we have

$$
M(2 \lambda) \leq c M(\lambda) .
$$

In the class of differentiable convex functions the $\Delta_{2}$-condition is equivalent to

$$
\lambda M^{\prime}(\lambda) \leq \widetilde{c} M(\lambda) \quad \text { for all } \lambda>0,
$$

with the constant $\widetilde{c}$ independent of $\lambda$ (see e.g. [35, Theorem 4.1]).

We will need the following property of modulars (see [35, formula (9.21)]):

$$
\int_{\mathbb{R}_{+}} M\left(\frac{f(x)}{\|f\|_{L_{\mu}^{M}}}\right) d \mu(x) \leq 1 .
$$

If $M$ satisfies the $\Delta_{2}$-condition, then (2.2) becomes an equality.

The function $M_{1}$ is said to dominate $M_{2}$ if there exist two positive constants $K_{1}, K_{2}$ such that $M_{2}(\lambda) \leq K_{1} M_{1}\left(K_{2} \lambda\right)$ for every $\lambda>0$. In that case we have

$$
\|\cdot\|_{L_{\mu}^{M_{2}}} \leq K\|\cdot\|_{L_{\mu}^{M_{1}}} \quad \text { with } \quad K=K_{2}\left(K_{1}+1\right) .
$$

Functions $M_{1}$ and $M_{2}$ are called equivalent when $M_{2}$ dominates $M_{1}$ and $M_{1}$ dominates $M_{2}$. In particular, equivalent $N$-functions give rise to equivalent Luxemburg norms. 
On the set $\mathcal{L}_{\mu}^{M}=\left\{u\right.$ measurable $\left.: \int M(|u|) d \mu<\infty\right\}$, one introduces the so-called dual norm:

$$
\begin{aligned}
& \|u\|_{L_{\mu}^{(M)}}=\sup \left\{\int_{\mathbb{R}_{+}} u(x) v(x) d \mu(x):\right. \\
& \left.v \in L_{\mu}^{M^{*}}, \int_{\mathbb{R}_{+}} M^{*}(|v(x)|) d \mu(x) \leq 1\right\} .
\end{aligned}
$$

The advantage of this norm is the Hölder-type inequality:

$$
\int_{\mathbb{R}_{+}} f \cdot g d \mu \leq\|f\|_{L_{\mu}^{(M)}}\|g\|_{L_{\mu}^{\left(M^{*}\right)}} \quad \text { for } f \in L_{\mu}^{M}, g \in L_{\mu}^{M^{*}} .
$$

If $M$ satisfies the $\Delta_{2}$-condition, then $\mathcal{L}_{\mu}^{M}=L_{\mu}^{M}$, and in general, the Orlicz space $L_{\mu}^{M}$ is the completion of $\mathcal{L}_{\mu}^{M}$ in the dual norm. The Luxemburg norm and the dual norm are equivalent:

$$
\|u\|_{L_{\mu}^{(M)}} \leq\|u\|_{L_{\mu}^{M}} \leq 2\|u\|_{L_{\mu}^{(M)}} .
$$

Assumptions. Throughout the paper we assume:

(M) $M:[0, \infty) \rightarrow[0, \infty)$ is a differentiable $N$-function, i.e. $M$ is convex, $M(0)=M_{+}^{\prime}(0)=0, M(\lambda) / \lambda \rightarrow \infty$ as $\lambda \rightarrow \infty$, and moreover

$$
d_{M} \frac{M(\lambda)}{\lambda} \leq M^{\prime}(\lambda) \leq D_{M} \frac{M(\lambda)}{\lambda} \quad \text { for every } \lambda>0
$$

where $D_{M} \geq d_{M} \geq 1$.

( $\mu$ ) $\mu$ is a Radon measure on $\mathbb{R}_{+}$, absolutely continuous with respect to the Lebesgue measure, and $\mu(d r)=e^{-\varphi(r)}$, where $\varphi \in C^{2}\left(\mathbb{R}_{+}\right)$and $\varphi^{\prime}$ never vanishes,

$(\omega) \quad \omega:(0, \infty) \rightarrow[0, \infty)$ is a $C^{1}$-function.

REMARK 2.1. The last inequality in (2.6) implies that $M$ satisfies the $\Delta_{2}$ condition ( $\operatorname{see}(2.1))$. The condition $d_{M}>1$ is equivalent to the $\Delta_{2}$-condition for $M^{*}$ (see e.g. [35, Theorem 4.3] or [30, Proposition 4.1]). Moreover, for any $N$-function $M$, the left-hand inequality in (2.6) holds with $d_{M}=1$.

If $d_{M}$ and $D_{M}$ are the best possible constants in (2.6) they obey the definition of the Simonenko lower and upper indices of $M$ and are related to Boyd indices of $L^{M}\left(\mathbb{R}^{n}, \mu\right)$ (see [7], [52] for definitions, and [18], [24], [58] for discussion on those and other indices of Orlicz spaces).

Notation. Before we proceed, we need to introduce the following quantities. We set

$$
\begin{aligned}
& \Omega:=\left\{r \in \mathbb{R}_{+}: \omega(r) u(r) \neq 0\right\}, \\
& F:=\left\{r \in \mathbb{R}_{+}: \omega(r) \neq 0, \omega^{\prime}(r) \varphi^{\prime}(r)>0\right\}, \\
& G:=\left\{r \in \mathbb{R}_{+}: \omega(r) \neq 0, \omega^{\prime}(r) \varphi^{\prime}(r)<0\right\} .
\end{aligned}
$$


Then we define

$$
\begin{aligned}
b_{1}(r, \omega, \varphi, M) & :=\left(1+\frac{\varphi^{\prime \prime}(r)}{\left(\varphi^{\prime}(r)\right)^{2}}-\frac{\omega^{\prime}(r)}{\omega(r) \varphi^{\prime}(r)}\left[d_{M} \chi_{G}(r)+D_{M} \chi_{F}(r)\right]\right), \\
b_{1} & :=b_{1}(\omega, \varphi, M):=\inf \left\{b_{1}(r, \omega, \varphi, M): r \in \mathbb{R}_{+}\right\}, \\
b_{2}(r, \omega, \varphi, M) & :=\left(-1-\frac{\varphi^{\prime \prime}(r)}{\left(\varphi^{\prime}(r)\right)^{2}}+\frac{\omega^{\prime}(r)}{\omega(r) \varphi^{\prime}(r)}\left[d_{M} \chi_{F}(r)+D_{M} \chi_{G}(r)\right]\right), \\
b_{2} & :=b_{2}(\omega, \varphi, M):=\inf \left\{b_{2}(r, \omega, \varphi, M): r \in \mathbb{R}_{+}\right\}, \\
L=L(\omega, \varphi) & :=\sup \left\{\frac{\omega(r)}{\left|\varphi^{\prime}(r)\right|}: r \in(0, \infty), \varphi^{\prime}(r) \neq 0\right\} .
\end{aligned}
$$

We use the conventions $\sup \emptyset=-\infty, \inf \emptyset=+\infty, c / \infty=0 ;$ and $f \chi_{A}$ is the function $f$ extended by 0 outside $A$.

2.2. Main results. Our area of interest will be those triples $(M, \varphi, \omega)$ for which either

(B1) $b_{1}>0, L<\infty$, or

(B2) $b_{2}>0, L<\infty$.

We will deal with the following function:

$$
h^{u}(r)=h^{(u, \omega, \varphi, M)}(r):=\frac{1}{\varphi^{\prime}(r)} M(\omega(r)|u(r)|),
$$

which is well defined since $\varphi^{\prime}(r)$ is never zero.

Let us introduce the following two classes of functions:

$$
\begin{aligned}
& \mathcal{R}_{(\omega, \varphi, M)}^{+}:=\left\{u \in W_{\mathrm{loc}}^{1,1}\left(\mathbb{R}_{+}\right)\right.: \\
& \lim _{n \rightarrow \infty}\left(h^{u}\left(R_{n}\right) e^{-\varphi\left(R_{n}\right)}-h^{u}\left(s_{n}\right) e^{-\varphi\left(s_{n}\right)}\right) \geq 0 \\
&\text { for some sequences } \left.s_{n} \rightarrow 0, R_{n} \rightarrow \infty\right\}, \\
& \mathcal{R}_{(\omega, \varphi, M)}^{-}:=\left\{u \in W_{\mathrm{loc}}^{1,1}\left(\mathbb{R}_{+}\right):\right. \\
& \lim _{n \rightarrow \infty}\left(h^{u}\left(R_{n}\right) e^{-\varphi\left(R_{n}\right)}-h^{u}\left(s_{n}\right) e^{-\varphi\left(s_{n}\right)}\right) \leq 0 \\
& \\
&\left.\quad \text { for some sequences } s_{n} \rightarrow 0, R_{n} \rightarrow \infty\right\},
\end{aligned}
$$

where the limits may be infinite. For simplicity we will usually omit $(\omega, \varphi, M)$ from the notation.

Note that both sets $\mathcal{R}^{+}$and $\mathcal{R}^{-}$contain the set of compactly supported $W^{1,1}$ functions and that they sum up to the whole $W_{\text {loc }}^{1,1}\left(\mathbb{R}_{+}\right)$.

Our main result reads as follows.

Theorem 2.1. Suppose that $M, \varphi, \omega$ satisfy $(\mathrm{M}),(\mu),(\omega)$ and $(\mathrm{B} 1)$ (respectively (B2)). Then

$$
\left.\int_{\mathbb{R}_{+}} M(\omega(r))|u(r)|\right) \mu(d r) \leq C \int_{\mathbb{R}_{+}} M\left(\left|u^{\prime}(r)\right|\right) \mu(d r)
$$


for every $u \in \mathcal{R}_{(\omega, \varphi, M)}^{+}$(resp. $\left.u \in \mathcal{R}_{(\omega, \varphi, M)}^{-}\right)$, where $C=c\left(L D_{M}^{2} / b_{1} d_{M}\right)$ (resp. $\left.C=c\left(L D_{M}^{2} / b_{2} d_{M}\right)\right)$ with $c(x)=\max \left(x^{d_{M}}, x^{D_{M}}\right)$.

As a direct consequence we also obtain the following theorem.

Theorem 2.2. Suppose that $M, \varphi, \omega$ satisfy $(\mathrm{M}),(\mu),(\omega)$ and $(\mathrm{B} 1)$ (respectively (B2)). Then

$$
\|\omega u\|_{L_{\mu}^{M}} \leq \widetilde{C}\left\|u^{\prime}\right\|_{L_{\mu}^{M}}
$$

for every $u \in \mathcal{R}_{(\omega, \varphi, M)}^{+}\left(\right.$resp. $\left.u \in \mathcal{R}_{(\omega, \varphi, M)}^{-}\right)$such that for every $\lambda>0, \lambda u \in$ $\mathcal{R}_{(\omega, \varphi, M)}^{+}\left(\right.$resp. $\left.\lambda u \in \mathcal{R}_{(\omega, \varphi, M)}^{-}\right)$as well, and where $\widetilde{C}=c\left(L D_{M}^{2} / b_{1} d_{M}\right)+1$ (resp. $\left.\widetilde{C}=c\left(L D_{M}^{2} / b_{2} d_{M}\right)+1\right)$ with $c(x)=\max \left(x^{d_{M}}, x^{D_{M}}\right)$.

\section{PARTICULAR CASES}

The main goal of this section is to illustrate Theorem 2.1 in various contexts. First we discuss the case when $M(\lambda)=\lambda^{p}$ (Subsection 3.1). Then we turn to general $M$ (Subsection 3.2). Finally, in Subsection 3.3, we restrict ourselves to weights $\omega=\left|\varphi^{\prime}\right|$.

3.1. Inequalities in the $L^{p}$ setting. When $M(\lambda)=\lambda^{p}$ with $p>1$, our conditions get simpler. In particular, $d_{M}=D_{M}=p$, and since $\varphi^{\prime}$ is assumed to be nonzero everywhere, we have

$$
b_{1}(r, \varphi, \omega)=1+\frac{\varphi^{\prime \prime}(r)}{\left(\varphi^{\prime}(r)\right)^{2}}-p \frac{\omega^{\prime}(r)}{\omega(r) \varphi^{\prime}(r)} \chi_{\{\omega(r) \neq 0\}}(r)=-b_{2}(r, \varphi, \omega) .
$$

It follows that our theorem yields results when $L<\infty$ and either $\inf _{r>0} b_{1}(r, \varphi, \omega)>0$, or $\sup _{r>0} b_{1}(r, \varphi, \omega)<0$.

3.1.1. Classical Hardy inequalities. As the first example illustrating our methods, we get the classical Hardy inequality (see e.g. [26, Theorem 330] for the classical source, Theorem 5.2 in [36], or [38] for the statement, historical framework and discussion).

Theorem 3.1. Let $1<p<\infty$ and $\alpha \neq p-1$. Suppose that $u=u(t)$ is an absolutely continuous function in $(0, \infty)$ such that $\int_{0}^{\infty}\left|u^{\prime}(t)\right|^{p} t^{\alpha} d t<\infty$, and let

$$
\begin{array}{ll}
u^{+}(0):=\lim _{t \rightarrow 0} u(t)=0 & \text { for } \alpha<p-1, \\
u(\infty):=\lim _{t \rightarrow \infty} u(t)=0 & \text { for } \alpha>p-1 .
\end{array}
$$

Then

$$
\int_{0}^{\infty}|u(t)|^{p} t^{\alpha-p} d t \leq C \int_{0}^{\infty}\left|u^{\prime}(t)\right|^{p} t^{\alpha} d t
$$

where $C=(p /|\alpha-p+1|)^{p}$. 
We consider the case $\alpha \neq 0$. Let us explain how this theorem follows from our results. Setting

$$
M(r)=r^{p}, \quad \mu(d r)=r^{\alpha} d r=\exp (\alpha \ln r), \quad \omega(r)=1 / r,
$$

we have

$$
\begin{gathered}
\varphi(r)=-\alpha \ln r, \quad \varphi^{\prime}(r)=-\alpha / r, \quad \varphi^{\prime \prime}(r)=\alpha / r^{2}, \\
\omega^{\prime}(r)=-1 / r^{2}, \quad \omega^{\prime}(r) \varphi^{\prime}(r)=\alpha / r^{2}>0, \quad d_{M}=D_{M}=p .
\end{gathered}
$$

By a direct check we see that

$$
b_{1}=\frac{\alpha-(p-1)}{\alpha}, \quad b_{2}=\frac{(p-1)-\alpha}{\alpha}, \quad L=\frac{1}{|\alpha|} .
$$

Therefore for $\alpha>p-1$ and for $\alpha<0$ we have $b_{1}>0$, while for $0<\alpha<p-1$ we have $b_{2}>0$. In either case $\varphi^{\prime}$ never vanishes. The constant $C$ in (2.13) is equal to $(p /|\alpha-(p-1)|)^{p}$, which coincides with the classical statement.

The only thing that remains to be checked is that any function $u$ as in the statement of Theorem 3.1 for which the right hand side in (3.1) is finite belongs to $\mathcal{R}^{+}$when $\alpha>p-1$ or $\alpha<0$, and to $\mathcal{R}^{-}$when $0<\alpha<p-1$. We will use standard arguments (see e.g. [36, proof of Theorem 5.2]).

First suppose that $\alpha>p-1$, and let $u$ be as in the assumptions of Theorem 3.1. Then for any $t>0$ we define $U(t):=\int_{t}^{\infty}|u(\tau)| d \tau$. One has

$$
\begin{aligned}
U(t) & =\int_{t}^{\infty}\left|u^{\prime}(\tau)\right| d \tau \leq \int_{t}^{\infty}\left|u^{\prime}(\tau)\right| \tau^{\alpha / p} \tau^{-\alpha / p} d \tau \\
& \leq\left(\int_{t}^{\infty}\left|u^{\prime}(\tau)\right|^{p} \tau^{\alpha} d \tau\right)^{1 / p}\left(\int_{t}^{\infty} \tau^{-\alpha /(p-1)} d \tau\right)^{(p-1) / p} \\
& =\left(\frac{p-1}{\alpha-(p-1)}\right)^{(p-1) / p}\left(\int_{t}^{\infty}\left|u^{\prime}(\tau)\right|^{p} \tau^{\alpha} d \tau\right)^{1 / p} t^{-(\alpha-(p-1)) / p}<\infty .
\end{aligned}
$$

From this chain of inequalities and the condition $\int_{0}^{\infty}\left|u^{\prime}(\tau)\right|^{p} \tau^{\alpha} d \tau<\infty$ we infer not only that $u \in W_{\text {loc }}^{1,1}(0, \infty)$ and that $U$ is well defined, but also that $\lim _{R \rightarrow \infty} U(R)^{p} R^{\alpha-(p-1)}=0$. Indeed, taking into account that $\lim _{t \rightarrow \infty} u(t)=0$, we have

$$
|u(t)|=\left|\int_{t}^{\infty} u^{\prime}(\tau) d \tau\right| \leq \int_{t}^{\infty}\left|u^{\prime}(\tau)\right| d \tau=U(t),
$$

and therefore $\lim _{R \rightarrow \infty}|u(R)|^{p} R^{\alpha-(p-1)}=0$ as well.

Recall now the formulas defining the class $\mathcal{R}^{+}$. The function $h(r) e^{-\varphi(r)}$ appearing there is now equal to $-\alpha^{-1}|u(r)|^{p} r^{\alpha-(p-1)}$, vanishing for $r$ tending to infinity. Therefore $u \in \mathcal{R}^{+}$. 
When $\alpha<p-1$, we proceed similarly, but now we take $U(t):=\int_{0}^{t}\left|u^{\prime}(\tau)\right| d \tau$. Again, $U$ is well defined and $\lim _{r \rightarrow 0} U(r)^{p} r^{\alpha-(p-1)}=0$, and since now $|u(r)| \leq U(r)$ as well, this yields $u \in \mathcal{R}^{-}$. We are done.

3.1.2. General approach within $L^{p}$-spaces. The following result is considered classical (see e.g. [44, Theorem 1 of Section 1.3.1]).

Theorem 3.2. Let $\mu, \nu$ be nonnegative Borel measures on $(0, \infty)$, let $\nu^{*}$ be the absolutely continuous part of $\nu$, and $1 \leq p \leq q \leq \infty$. Then

$$
\left(\int_{\mathbb{R}_{+}}\left|\int_{0}^{t} f(\tau) d \tau\right|^{q} d \mu(t)\right)^{1 / q} \leq C\left(\int_{\mathbb{R}_{+}}|f(t)|^{p} d \nu(t)\right)^{1 / p}
$$

for all locally integrable functions $f$ if and only if

$$
B:=\sup _{r>0}(\mu[r, \infty))^{1 / q}\left(\int_{0}^{r}\left(\frac{d \nu^{*}}{d \tau}\right)^{-1 /(p-1)} d \tau\right)^{(p-1) / p}<\infty .
$$

The case $p=q \geq 1$ is due to Muckenhoupt [46]. Extensions to general $p, q$ were proven by Mazya and Rozin ([44, Theorem 1 of Section 1.3.1]), Bradley [8] and Kokilashvili [33]. Some other generalizations (admitting also $p, q$ below 1) were obtained by Sinnamon [53], Sawyer [51], Bloom and Kerman [6], Stepanov [54] and others.

Observe that inequality (2.13) corresponding to $\omega(r)=r$ is a particular case of (3.2) when one takes $\nu(d r)=e^{-\varphi(r)} d r, p=q, \mu(d r)=r^{p} e^{-\varphi(r)} d r$, but only for the representative $u(t)=\int_{0}^{t} u^{\prime}(\tau) d \tau$. In particular, $u^{+}(0)=0$, which we have not required (Theorem 3.1 shows that in general the condition $u^{+}(0)=0$ may not hold). In this case (3.2) reads

$$
\int_{\mathbb{R}_{+}}|u(\tau) \omega(\tau)|^{p} d \nu(\tau) \leq C \int_{\mathbb{R}_{+}}\left|u^{\prime}(\tau)\right|^{p} d \nu(\tau),
$$

and $u(t)=\int_{0}^{t} u^{\prime}(s) d s, u$ is an absolutely continuous function. The condition $B<\infty$ is equivalent to

$$
\sup _{r>0}\left(\int_{r}^{\infty} x^{p} \exp (-\varphi(x)) d x\right)\left(\int_{0}^{r} \exp \left(\frac{\varphi(x)}{p-1}\right) d x\right)^{p-1}<\infty .
$$

It is of different nature than our conditions (B1) and (B2) and is usually not easy to handle. But since $B<\infty$ is equivalent to the inequality (3.2) holding for all $u$ in the set $\mathcal{H}$ of Hardy transforms (see (1.3)), our assumptions can serve as a tool towards verifying $B<\infty$.

We may as well deal with the set $\mathcal{H}^{*}$ of conjugate Hardy transforms $u=-\int_{t}^{\infty} f(\tau) d \tau$ (see (1.3)) instead of $u=\int_{0}^{t} f(\tau) d \tau \in \mathcal{H}$ as illustrated in Theorem 3.1, the case $\alpha>p-1$. 
It may happen that inequalities (3.2) do not hold in general on the whole set $\mathcal{H}$, but they do hold on some smaller sets. These are the sets $\mathcal{R}^{+}, \mathcal{R}^{-}$ defined by (2.11) and (2.12).

3.2. Results in Orlicz spaces. The papers of Maz'ya, Bloom-Kerman and Lai $[44,6,40,41,42,43,27]$ are concerned with inequalities of the form

$$
Q^{-1}\left(\int_{\mathbb{R}_{+}} Q(\omega(x)|T f(x)|) r(x) d x\right) \leq P^{-1}\left(\int_{\mathbb{R}_{+}} P(C \varrho(x)|f(x)|) v(x) d x\right),
$$

where $T f$ is the Hardy-type operator

$$
T f(x)=\int_{0}^{x} K(x, y) f(y) d y, \quad x>0,
$$

with a suitable kernel $K$. The case $K=1$, corresponding to the classical Hardy operator, is also covered. $P$ and $Q$ are assumed to be nondecreasing functions on $\mathbb{R}_{+}$satisfying

$$
\lim _{t \rightarrow 0^{+}} P(t)=\lim _{t \rightarrow 0^{+}} Q(t)=0, \quad \lim _{t \rightarrow \infty} P(t)=\lim _{t \rightarrow \infty} Q(t)=\infty .
$$

Bloom and Kerman proved in [6] that within the class of modular functions $P$ and $Q$ satisfying the following domination property:

- there exists a constant $\eta>0$ for which $\sum Q P^{-1}\left(a_{j}\right) \leq Q P^{-1}\left(\eta \sum a_{j}\right)$ whenever $\left\{a_{j}\right\}$ is any nonnegative sequence,

(3.3) is equivalent to the conditions

$$
\left\{\begin{array}{l}
\int_{0}^{y} P^{*}\left(\frac{G(\varepsilon, y) K(y, x)}{B \varepsilon v(x) \varrho(x)}\right) v(x) d x \leq G(\varepsilon, y)<\infty, \\
\int_{0}^{y} P^{*}\left(\frac{H(\varepsilon, y)}{B \varepsilon v(x) \varrho(x)}\right) v(x) d x \leq H(\varepsilon, y)<\infty
\end{array}\right.
$$

holding for all $y>0$ and $\varepsilon>0$, where $P^{*}$ is the Legendre transform of $P$,

$$
\begin{aligned}
& G(\varepsilon, y)=P Q^{-1}\left(\int_{y}^{\infty} Q(\varepsilon \omega(x)) r(x) d x\right), \\
& H(\varepsilon, y)=P Q^{-1}\left(\int_{y}^{\infty} Q(\varepsilon \omega(x) K(x, y)) r(x) d x\right),
\end{aligned}
$$

and $B>0$ is a constant.

In our particular case: $P=Q(=M)$ with $M$ satisfying the $\Delta_{2}$-condition, $r=v=\exp (-\varphi(x)), K \equiv 1, \varrho \equiv 1$, inequality (3.3) reduces to 


$$
\int_{\mathbb{R}_{+}} M(\omega(x) T f(x)) \exp (-\varphi(x)) d x \leq C\left(\int_{\mathbb{R}_{+}} M(f(x)) \exp (-\varphi(x)) d x\right),
$$

which is an inequality of the type we are dealing with.

In this case conditions (3.4) simplify to

$$
\int_{0}^{y} M^{*}\left(\frac{G(\varepsilon, y)}{B \varepsilon \exp (-\varphi(x))}\right) \exp (-\varphi(x)) d x \leq G(\varepsilon, y)<\infty
$$

for all $y>0$ and $\varepsilon>0$, where

$$
G(\varepsilon, y)=\int_{y}^{\infty} M(\varepsilon \omega(x)) \exp (-\varphi(x)) d x,
$$

and $B>0$ is a constant. A further generalization of (3.3), without the restriction $P \ll Q$, can be found in Lai's paper [43].

Condition (3.6) as well as Lai's condition are not easily implemented: in practice, for given $\omega, \varphi, M$, it is usually hard to see whether (3.6) holds or not. Conditions (B1) and (B2) are much simpler. When they are satisfied, and when we know that the Hardy operator $T f(x)$ (or the dual Hardy operator $T^{*} f(x)=\int_{x}^{\infty} f(\tau) d \tau$, see [43, last remark on page 671]) belongs to the set $\mathcal{R}^{-}$or $\mathcal{R}^{+}$, then inequality (3.5) is just the statement of Theorem 2.1.

3.3. Special choice of weights. The case of $\omega=\left|\varphi^{\prime}\right|$. Another case that substantially simplifies the approach is that of $\omega=\left|\varphi^{\prime}\right|$ (in fact this was used in the proof of the classical Hardy inequality). Since we require $\varphi$ to be $C^{1}$ with nonzero derivative, $\varphi^{\prime}$ is either always positive, or always negative.

This time, we have

$$
\begin{aligned}
& b_{1}\left(r,\left|\varphi^{\prime}\right|, \varphi, M\right)= \begin{cases}1+\left(1-d_{M}\right) \frac{\varphi^{\prime \prime}(r)}{\varphi^{\prime}(r)^{2}} & \text { if } \varphi^{\prime \prime}(r) \leq 0, \\
1+\left(1-D_{M}\right) \frac{\varphi^{\prime \prime}(r)}{\varphi^{\prime}(r)^{2}} & \text { if } \varphi^{\prime \prime}(r) \geq 0,\end{cases} \\
& b_{2}\left(r,\left|\varphi^{\prime}\right|, \varphi, M\right)= \begin{cases}-1+\left(D_{M}-1\right) \frac{\varphi^{\prime \prime}(r)}{\left(\varphi^{\prime}(r)\right)^{2}} & \text { if } \varphi^{\prime \prime}(r) \leq 0, \\
-1+\left(d_{M}-1\right) \frac{\varphi^{\prime \prime}(r)}{\left(\varphi^{\prime}(r)\right)^{2}} & \text { if } \varphi^{\prime \prime}(r) \geq 0,\end{cases} \\
& L=1 \text {. }
\end{aligned}
$$

In particular (as $D_{M} \geq d_{M} \geq 1$ ), we get

$$
b_{1}>0 \quad \text { if and only if } \sup _{r>0} \frac{\varphi^{\prime \prime}(r)}{\left(\varphi^{\prime}(r)\right)^{2}}<\frac{1}{D_{M}-1}=: \widetilde{D}_{M},
$$


$b_{2}>0$ if and only if $\inf _{r>0} \frac{\varphi^{\prime \prime}(r)}{\left(\varphi^{\prime}(r)\right)^{2}}>\frac{1}{d_{M}-1}=: \widetilde{d}_{M}$.

This leads to the following conclusion, which is of independent interest.

Corollary 3.1. Assume that conditions $(\mathrm{M})$ and $(\mu)$ are satisfied, and either $\sup _{r>0} \frac{\varphi^{\prime \prime}(r)}{\left(\varphi^{\prime}(r)\right)^{2}}<\widetilde{D}_{M} \quad$ or $\inf _{r>0} \frac{\varphi^{\prime \prime}(r)}{\left(\varphi^{\prime}(r)\right)^{2}}>\widetilde{d}_{M}$.

Then

$$
\int_{\mathbb{R}_{+}} M\left(\left|\varphi^{\prime}(r)\right||u(r)|\right) \exp (-\varphi(r)) d r \leq C \int_{\mathbb{R}_{+}} M\left(\left|u^{\prime}(r)\right|\right) \exp (-\varphi(r)) d r
$$

and

$$
\left\|\varphi^{\prime} u\right\|_{L_{\mu}^{M}} \leq \widetilde{C}\left\|u^{\prime}\right\|_{L_{\mu}^{M}}
$$

for every $u \in \mathcal{R}_{\left(\left|\varphi^{\prime}\right|, \varphi, M\right)}^{+}$with $C=c\left(D_{M}^{2} / b_{1} d_{M}\right), \widetilde{C}=c\left(D_{M}^{2} / b_{1} d_{M}\right)+1$ in the first case, and for every $u \in \mathcal{R}_{\left(\left|\varphi^{\prime}\right|, \varphi, M\right)}^{-}$with $C=c\left(D_{M}^{2} / b_{2} d_{M}\right)$, $\widetilde{C}=c\left(D_{M}^{2} / b_{2} d_{M}\right)+1$ in the other case, where $c(r)=\max \left(r^{d_{M}}, r^{D_{M}}\right)$.

EXAMPLE 3.1 (classical inequalities). To illustrate this corollary we consider again $M(\lambda)=\lambda^{p}, p>1, \varphi(\lambda)=-\alpha \ln r$ as in Section 3.1.1. In this case

$$
d_{M}=D_{M}=p, \quad \frac{\varphi^{\prime \prime}}{\left(\varphi^{\prime}\right)^{2}} \equiv \frac{1}{\alpha} .
$$

We have

$$
\begin{array}{ll}
\frac{1}{\alpha} \equiv \sup _{r>0} \frac{\varphi^{\prime \prime}(r)}{\left(\varphi^{\prime}(r)\right)^{2}}<\frac{1}{p-1}=\widetilde{D}_{M} \quad \text { for } \alpha \in(-\infty, 0) \cup(p-1, \infty), \\
\frac{1}{\alpha} \equiv \inf _{r>0} \frac{\varphi^{\prime \prime}(r)}{\left(\varphi^{\prime}(r)\right)^{2}}>\frac{1}{p-1}=\widetilde{d}_{M} \quad \text { for } \alpha \in(0, p-1) .
\end{array}
$$

Therefore the classical Hardy inequality follows from Corollary 3.1 as well.

EXAMPLE 3.2 (Hardy inequalities with logarithmic-type weights). As another illustration we show what inequality can be obtained for the measures $\mu(d r)=r^{\alpha}(\ln (1+r))^{\beta} d r$ with $\alpha, \beta>0$.

In this case we have

$$
\begin{aligned}
\varphi(r) & =: \varphi_{\alpha, \beta}(r)=-\alpha \ln (r)-\beta \ln \ln (1+r), \\
\varphi^{\prime}(r) & =-\frac{\alpha}{r}-\frac{\beta}{\ln (1+r)} \frac{1}{1+r} \\
\varphi^{\prime \prime}(r) & =\frac{\alpha}{r^{2}}+\frac{\beta}{(1+r)^{2}} \frac{1}{\ln (1+r)}\left(1+\frac{1}{\ln (1+r)}\right) .
\end{aligned}
$$


Choose $\omega(r)=\left|\varphi^{\prime}(r)\right| ;$ then

$$
b_{1}(r)=1+\left(1-D_{M}\right) \frac{\varphi^{\prime \prime}(r)}{\left(\varphi^{\prime}(r)\right)^{2}},
$$

and so $b_{1}=1-\left(D_{M}-1\right) \sup _{r>0} \varphi^{\prime \prime}(r) /\left(\varphi^{\prime}(r)\right)^{2}$. As $\varphi^{\prime}(r)$ is of order $1 / r$ and $\varphi^{\prime \prime}(r)$ is of order $1 / r^{2}$ on $\mathbb{R}_{+}$, the supremum involved is finite. Set

$$
s_{\alpha, \beta}=\sup _{r>0} \frac{\varphi^{\prime \prime}(r)}{\left(\varphi^{\prime}(r)\right)^{2}} .
$$

Then

$$
b_{1}>0 \Leftrightarrow D_{M}<1+\frac{1}{s_{\alpha, \beta}} .
$$

As $1 / r \sim \omega(r)$, we arrive at the following.

TheOREM 3.3. Suppose $\alpha, \beta>0$ and let $M$ be an $N$-function satisfying (M) such that $D_{M}<1+1 / s_{\alpha, \beta}$, where $s_{\alpha, \beta}$ is given by (3.7). Then there exists a constant $C>0$ such that

$$
\int_{\mathbb{R}_{+}} M\left(\frac{u(r)}{r}\right) r^{\alpha}(\ln (1+r))^{\beta} d r \leq C \int_{\mathbb{R}_{+}} M\left(\left|u^{\prime}(r)\right|\right) r^{\alpha}(\ln (1+r))^{\beta} d r
$$

for all $u \in \mathcal{R}^{+}\left(\left|\varphi^{\prime}\right|, \varphi, M\right) \supset C_{0}^{1}\left(\mathbb{R}_{+}\right)$.

A similar analysis can be performed for negative $\alpha$ or $\beta$.

How to easily verify whether $u \in \mathcal{R}^{+}$or $u \in \mathcal{R}^{-}$will be shown in Subsection 5.1.

\section{PROOFS OF THEOREMS 2.1 AND 2.2}

Before we pass to the actual proofs, let us formulate two easy lemmas concerning Young functions. Although the lemmas may be known to specialists (see e.g. [24], [52] and [18] for related results), for the reader's convenience we supply the proofs.

Lemma 4.1. Let $M$ be a differentiable $N$-function.

(i) Suppose that there exists a constant $D_{M} \geq 1$ such that

$$
M^{\prime}(r) \leq D_{M} \frac{M(r)}{r} \quad \text { for every } r>0 .
$$

Then for all $r>0$ and $\lambda \geq 1$,

$$
M(\lambda r) \leq \lambda^{D_{M}} M(r) .
$$

(ii) Suppose that there exists a constant $d_{M} \geq 1$ such that

$$
d_{M} \frac{M(r)}{r} \leq M^{\prime}(r) \quad \text { for every } r>0 .
$$

Then for all $r>0$ and $\lambda \leq 1$,

$$
M(\lambda r) \leq \lambda^{d_{M}} M(r) .
$$


(iii) Suppose that there exist constants $1 \leq d_{M} \leq D_{M}$ such that

$$
d_{M} \frac{M(r)}{r} \leq M^{\prime}(r) \leq D_{M} \frac{M(r)}{r} \quad \text { for every } r>0 .
$$

Then for all $r, \lambda>0$,

$$
M(\lambda r) \leq \max \left(\lambda^{d_{M}}, \lambda^{D_{M}}\right) M(r)=: c(\lambda) M(r) .
$$

We recall Remark 2.1 for interpretation of the constants $d_{M}, D_{M}$.

Proof. We only prove (i); (ii) is proven analogously, while (iii) is their direct consequence.

From (4.1) we get $M^{\prime}(r) / M(r) \leq D_{M} / r$, and further, for any $r>0$ and $\lambda>1$,

$$
\int_{r}^{\lambda r} \frac{M^{\prime}(t)}{M(t)} d t \leq \int_{r}^{\lambda r} \frac{D_{M}}{t} d t,
$$

which after integrating gives $[\ln M(t)]_{r}^{\lambda r} \leq\left[\ln t^{D_{M}}\right]_{r}^{\lambda r}$, and further $M(\lambda r) \leq$ $\lambda^{D_{M}} M(r)$.

Lemma 4.2. Let $M$ be a differentiable $N$-function, and suppose that $1 \leq$ $d_{M} \leq D_{M}$ are two constants such that

$$
d_{M} \frac{M(r)}{r} \leq M^{\prime}(r) \leq D_{M} \frac{M(r)}{r} \quad \text { for every } r>0 .
$$

Then for all $r, s>0$,

$$
\frac{M(r)}{r} s \leq \frac{D_{M}-1}{d_{M}} M(r)+\frac{1}{d_{M}} M(s) .
$$

Proof. Using the Young inequality $r s \leq M^{*}(r)+M(s)$ together with (4.3) we have

$$
\frac{M(r)}{r} s \leq \frac{1}{d_{M}} M^{\prime}(r) s \leq \frac{M^{*}\left(M^{\prime}(r)\right)}{d_{M}}+\frac{M(s)}{d_{M}} .
$$

From the very definition of the conjugate function $M^{*}$ we have

$$
M(r)=r M^{\prime}(r)-M^{*}\left(M^{\prime}(r)\right),
$$

and so

$$
M^{*}\left(M^{\prime}(r)\right) \leq D_{M} M(r)-M(r)=\left(D_{M}-1\right) M(r) .
$$

Inserting this into (4.5) we get (4.4).

Proof of Theorem 2.1. Suppose $u \in \mathcal{R}_{(\omega, \varphi, M)}^{+}$(resp. $\left.u \in \mathcal{R}_{(\omega, \varphi, M)}^{-}\right)$. Choose $s_{n} \rightarrow 0$ and $R_{n} \rightarrow \infty$ as in (2.11) (resp. (2.12)). To abbreviate, 
we write

$$
\begin{array}{rlrl}
J:= & \left.\int_{0}^{\infty} M(\omega(r))|u(r)|\right) e^{-\varphi(r)} d r, & \left.J_{n}:=\int_{s_{n}}^{R_{n}} M(\omega(r))|u(r)|\right) e^{-\varphi(r)} d r, \\
H:=\int_{0}^{\infty} M\left(\left|u^{\prime}(r)\right|\right) e^{-\varphi(r)} d r, & H_{n}:=\int_{s_{n}}^{R_{n}} M\left(\left|u^{\prime}(r)\right|\right) e^{-\varphi(r)} d r .
\end{array}
$$

Let $h^{u}$ be given by (2.10). Under our assumptions, it is well defined for every $r>0$. Since $u \in W_{\text {loc }}^{1,1}\left(\mathbb{R}^{n}\right)$ and $M$ is locally Lipschitz, we infer that $h^{u} \in W_{\mathrm{loc}}^{1,1}\left(\mathbb{R}_{+}\right)$and

$$
\begin{aligned}
\left(h^{u}\right)^{\prime}(r)= & \frac{d}{d r}\left(\frac{1}{\varphi^{\prime}(r)}\right) M(\omega(r)|u(r)|) \\
& +\frac{1}{\varphi^{\prime}(r)} M^{\prime}(\omega(r)|u(r)|)\left(\omega^{\prime}(r)|u(r)|+\omega(r) u^{\prime}(r) \operatorname{sgn} u(r)\right),
\end{aligned}
$$

in the sense of distributions and almost everywhere, and $h$ is absolutely continuous on each interval $[s, R] \subseteq(0, \infty)$ (see e.g. [44, Theorems 1 and 2 , Sec. 1.1.3]). Moreover, for $0<s<R<\infty$,

$$
\begin{aligned}
\int_{s}^{R}\left(h^{u}\right)^{\prime}(r) e^{-\varphi(r)} d r & =\left.h^{u}(r) e^{-\varphi(r)}\right|_{s} ^{R}+\int_{s}^{R} M(\omega(r)|u(r)|) e^{-\varphi(r)} d r \\
& =: \theta(R, s)+\int_{s}^{R} M(\omega(r)|u(r)|) e^{-\varphi(r)} d r,
\end{aligned}
$$

and so we have

$$
J_{n}=\int_{s_{n}}^{R_{n}}\left(h^{u}\right)^{\prime}(r) e^{-\varphi(r)} d r-\theta_{n}
$$

where $\theta_{n}:=\theta\left(R_{n}, s_{n}\right) \rightarrow \alpha \in[0, \infty]$ (resp. $\left.[-\infty, 0]\right)$.

Inserting (4.6) in (4.7) and using (2.7) yields, after some rearrangement,

$$
\begin{aligned}
J_{n}= & \int_{s_{n}}^{R_{n}}\left(-\frac{\varphi^{\prime \prime}(r)}{\left(\varphi^{\prime}(r)\right)^{2}}\right) M(\omega(r)|u(r)|) e^{-\varphi(r)} d r \\
& -\int_{s_{n}}^{R_{n}} \frac{1}{\left|\varphi^{\prime}(r)\right|} M^{\prime}(\omega(r)|u(r)|)\left|\omega^{\prime}(r)\right||u(r)| \chi_{G}(r) e^{-\varphi(r)} d r
\end{aligned}
$$

$$
\begin{array}{r}
+\int_{s_{n}}^{R_{n}} \frac{1}{\left|\varphi^{\prime}(r)\right|} M^{\prime}(\omega(r)|u(r)|)\left(\left|\omega^{\prime}(r)\right||u(r)| \chi_{F}(r)+\omega(r) u^{\prime}(r) \operatorname{sgn} u(r)\right) e^{-\varphi(r)} d r-\theta_{n} \\
=: I_{n}-I I_{n}+I I I_{n}-\theta_{n} .
\end{array}
$$


From now on, the proofs for the two cases: (B1) with $u \in \mathcal{R}^{+}$, and (B2) with $u \in \mathcal{R}^{-}$, differ slightly.

Case 1. Assume that (B1) is satisfied and $u \in \mathcal{R}^{+}$. Since we have $-M^{\prime}(\lambda) \leq-d_{M} M(\lambda) / \lambda$, it follows that

$$
\begin{aligned}
-I I_{n} & \leq-\int_{s_{n}}^{R_{n}} d_{M} M(\omega(r)|u(r)|) \frac{\left|\omega^{\prime}(r)\right|}{\omega(r)\left|\varphi^{\prime}(r)\right|} \chi_{G}(r) e^{-\varphi(r)} d r \\
& =\int_{s_{n}}^{R_{n}} d_{M} M(\omega(r)|u(r)|) \frac{\omega^{\prime}(r)}{\omega(r) \varphi^{\prime}(r)} \chi_{G}(r) e^{-\varphi(r)} d r=: I V_{n} .
\end{aligned}
$$

For $I I I_{n}$, we first estimate $u^{\prime}(r) \operatorname{sgn} u(r)$ by $\left|u^{\prime}(r)\right|$, and then use the inequality $M^{\prime}(\lambda) \leq D_{M} M(\lambda) / \lambda$. For every $r \in \Omega$ (see (2.7)) we have

$$
\begin{aligned}
& \left.M^{\prime}(\omega(r)|u(r)|)\left(\left|\omega^{\prime}(r)\right||u(r)|\right) \chi_{F}(r)+\omega(r) u^{\prime}(r) \operatorname{sgn} u^{\prime}(r)\right) \\
& \quad \leq D_{M}\left[M(\omega(r)|u(r)|) \frac{\left|\omega^{\prime}(r)\right|}{\omega(r)} \chi_{F}(r)+M(\omega(r)|u(r)|) \frac{\left|u^{\prime}(r)\right|}{|u(r)|}\right] \\
& \quad=: D_{M}\left[A_{1}(r)+A_{2}(r)\right] .
\end{aligned}
$$

This implies

$$
\text { (4.11) } \quad I I I_{n} \leq D_{M} \int_{\Omega \cap\left[s_{n}, R_{n}\right]} \frac{1}{\left|\varphi^{\prime}(r)\right|}\left[A_{1}(r)+A_{2}(r)\right] e^{-\varphi(r)} d r=: V_{n}+V I_{n},
$$

where

$$
\begin{aligned}
V_{n} & =\int_{\Omega \cap\left[s_{n}, R_{n}\right]} D_{M} \frac{\left|\omega^{\prime}(r)\right|}{\omega(r)\left|\varphi^{\prime}(r)\right|} \chi_{F}(r) M(\omega(r)|u(r)|) e^{-\varphi(r)} d r \\
& =\int_{\Omega \cap\left[s_{n}, R_{n}\right]} D_{M} \frac{\omega^{\prime}(r)}{\omega(r) \varphi^{\prime}(r)} \chi_{F}(r) M(\omega(r)|u(r)|) e^{-\varphi(r)} d r \\
V I_{n} & =\int_{\Omega \cap\left[s_{n}, R_{n}\right]} D_{M} \frac{\left|u^{\prime}(r)\right|}{|u(r)|\left|\varphi^{\prime}(r)\right|} M(\omega(r)|u(r)|) e^{-\varphi(r)} d r .
\end{aligned}
$$

The definition $(2.8)$ of the constant $b_{1}$ yields

$$
b_{1} J_{n} \leq J_{n}-I_{n}-I V_{n}-V_{n},
$$

and (4.8), (4.9), (4.11) give

$$
J_{n}=I_{n}-I I_{n}+I I I_{n}-\theta_{n} \leq I_{n}+I V_{n}+V_{n}+V I_{n}-\theta_{n} .
$$

Combining the two we get

$$
b_{1} J_{n} \leq V I_{n}-\theta_{n} .
$$

Consequently, since $b_{1}$ is assumed to be positive,

$$
J_{n} \leq \frac{D_{M}}{b_{1}} \int_{\Omega \cap\left[s_{n}, R_{n}\right]} \frac{A_{2}(r)}{\left|\varphi^{\prime}(r)\right|} e^{-\varphi(r)} d r-\frac{\theta_{n}}{b_{1}} .
$$


Now we use the estimates from Lemmas 4.1 and 4.2. For any $0<\delta \leq 1$ (or any $\delta>0$ when $M(\lambda)=\lambda^{p}$ ) and $r \in \Omega$,

$$
\begin{aligned}
A_{2}(r) & =\delta \omega(r) \frac{M(\omega(r)|u(r)|)}{\omega(r)|u(r)|} \frac{\left|u^{\prime}(r)\right|}{\delta} \\
& \leq \delta \omega(r)\left[\frac{D_{M}-1}{d_{M}} M(\omega(r)|u(r)|)+\frac{1}{d_{M}} M\left(\frac{\left|u^{\prime}(r)\right|}{\delta}\right)\right] \\
& \leq \delta \omega(r)\left[\frac{D_{M}-1}{d_{M}} M(\omega(r)|u(r)|)+\frac{1}{d_{M}} c\left(\frac{1}{\delta}\right) M\left(\left|u^{\prime}(r)\right|\right)\right]
\end{aligned}
$$

(Lemma 4.1 was used in the very last line).

Using now this estimate on $A_{2}(r)$, we obtain from (4.12)

$$
\begin{aligned}
J_{n} \leq & \frac{D_{M} \delta}{b_{1}}\left(\frac{D_{M}-1}{d_{M}} \int_{s_{n}}^{R_{n}} \frac{\omega(r)}{\left|\varphi^{\prime}(r)\right|} M(\omega(r)|u(r)|) e^{-\varphi(r)} d r\right. \\
& \left.+\frac{1}{d_{M}} c\left(\frac{1}{\delta}\right) \int_{s_{n}}^{R_{n}} \frac{\omega(r)}{\left|\varphi^{\prime}(r)\right|} M\left(\left|u^{\prime}(r)\right|\right) e^{-\varphi(r)} d r\right)-\frac{\theta_{n}}{b_{1}} \\
\leq & \frac{D_{M} L \delta}{b_{1}}\left(\frac{D_{M}-1}{d_{M}} J_{n}+\frac{1}{d_{M}} c\left(\frac{1}{\delta}\right) H_{n}\right)-\frac{\theta_{n}}{b_{1}},
\end{aligned}
$$

or, after rearranging,

$$
\frac{\theta_{n}}{b_{1}}+\left(1-\frac{D_{M} L \delta}{b_{1}} \frac{D_{M}-1}{d_{M}}\right) J_{n} \leq \frac{D_{M} L \delta}{b_{1} d_{M}} c\left(\frac{1}{\delta}\right) H_{n} .
$$

Choose now $\delta_{0}=\frac{b_{1} d_{M}}{L D_{M}^{2}}$ to obtain

$$
\frac{D_{M} \theta_{n}}{b_{1}}+J_{n} \leq c\left(\frac{1}{\delta_{0}}\right) H_{n} .
$$

Only now do we let $n \rightarrow \infty$. As the limits $\lim _{n \rightarrow \infty} J_{n}=J, \lim _{n \rightarrow \infty} H_{n}$ $=H$ and $\lim _{n \rightarrow \infty} \theta_{n}$ are all well defined and nonnegative (finite or not), this implies

$$
J \leq c\left(\frac{L D_{M}^{2}}{b_{1} d_{M}}\right) H
$$

and finishes the proof.

CASE 2. We now prove the statement under assumption (B2), for $u \in \mathcal{R}^{-}$. We start with an expression similar to (4.8), but the integrals are rearranged somewhat differently. 
This time we write

$$
\begin{aligned}
J_{n}= & \int_{s_{n}}^{R_{n}}\left(-\frac{\varphi^{\prime \prime}(r)}{\left(\varphi^{\prime}(r)\right)^{2}}\right) M(\omega(r)|u(r)|) e^{-\varphi(r)} d r \\
& +\int_{s_{n}}^{R_{n}} \frac{1}{\left|\varphi^{\prime}(r)\right|} M^{\prime}(\omega(r)|u(r)|)\left|\omega^{\prime}(r)\right||u(r)| \chi_{F}(r) e^{-\varphi(r)} d r
\end{aligned}
$$

$$
\begin{array}{r}
-\int_{s_{n}}^{R_{n}} \frac{1}{\left|\varphi^{\prime}(r)\right|} M^{\prime}(\omega(r)|u(r)|)\left(\left|\omega^{\prime}(r)\right||u(r)| \chi_{G}(r)+\omega(r) u^{\prime}(r) \operatorname{sgn} u(r)\right) e^{-\varphi(r)} d r-\theta_{n} \\
=: I_{n}+I I_{n}^{\prime}-I I I_{n}^{\prime}-\theta_{n} .
\end{array}
$$

As before, since $M^{\prime}(\lambda) \geq d_{M} M(\lambda) / \lambda$, we get

$$
I I_{n}^{\prime} \geq \int_{s_{n}}^{R_{n}} d_{M} M(\omega(r)|u(r)|) \frac{\omega^{\prime}(r)}{\omega(r) \varphi^{\prime}(r)} \chi_{F}(r) e^{-\varphi(r)} d r=: I V_{n}^{\prime}
$$

(one can omit the absolute values because $r \in F$ ). Furthermore, since $-M^{\prime}(\lambda) \geq-D_{M} M(\lambda) / \lambda$, for every $r \in \Omega$ we have

$$
\begin{array}{r}
-M^{\prime}(\omega(r)|u(r)|)\left(\left|\omega^{\prime}(r)\right||u(r)| \chi_{G}(r)+\omega(r)\left|u^{\prime}(r)\right|\right) \\
\geq-D_{M}\left[M(\omega(r)|u(r)|) \frac{\left|\omega^{\prime}(r)\right|}{\omega(r)} \chi_{G}(r)+M(\omega(r)|u(r)|) \frac{\left|u^{\prime}(r)\right|}{|u(r)|}\right] \\
=:-D_{M}\left[B(r)+A_{2}(r)\right],
\end{array}
$$

where $A_{2}(r)$ is as in (4.10). This last estimate combined with (4.13) implies

$$
-I I I_{n}^{\prime} \geq-D_{M} \int_{\Omega \cap\left[s_{n}, R_{n}\right]} \frac{1}{\left|\varphi^{\prime}(r)\right|}\left[B(r)+A_{2}(r)\right] e^{-\varphi(r)} d r=: V_{n}^{\prime}+V I_{n}^{\prime} .
$$

On the other hand,

$$
\begin{aligned}
b_{2} J_{n} & \leq I_{n}+I V_{n}^{\prime}+V_{n}^{\prime}-J_{n}, \\
J_{n} & \geq I_{n}+I I_{n}^{\prime}-I I I_{n}^{\prime}-\theta_{n} \geq I_{n}+I V_{n}^{\prime}+V_{n}^{\prime}+V I_{n}^{\prime}-\theta_{n} .
\end{aligned}
$$

We get $b_{2} J_{n} \leq-V I_{n}^{\prime}+\theta_{n}$, which leads to

$$
J_{n} \leq \frac{D_{M}}{b_{2}} \int_{\Omega \cap\left[s_{n}, R_{n}\right]} \frac{A_{2}(r)}{\left|\varphi^{\prime}(r)\right|} e^{-\varphi(r)} d r+\frac{\theta_{n}}{b_{2}} .
$$

Now the proof follows the lines of the first case, starting from (4.12), with $b_{2}$ replacing $b_{1}$ and $\theta_{n}$ replacing $-\theta_{n}$.

REMARK 4.1. Observe that for $M(\lambda)=\lambda^{p}$ we have $d_{M}=D_{M}=p$ and $c(x)=x^{p}$. Therefore under the assumptions of Theorem 2.1 the constant $C$ equals either $\left(L p / b_{1}\right)^{p}$ if $b_{1}>0$, or $\left(L p / b_{2}\right)^{p}$ if $b_{2}>0$. 
Proof of Theorem 2.2. Without loss of generality we can assume that $A=\left\|u^{\prime}\right\|_{L_{\mu}^{M}}$ is finite. Let us substitute $u_{A}:=u / A$ in (2.13). Then we get

$$
\int_{\mathbb{R}_{+}} \frac{M}{C}\left(\frac{\omega|u|}{A}\right) \exp (-\varphi) \leq 1,
$$

which implies $\|\omega u\|_{L_{\mu}^{M / C}} \leq A$. As the $N$-functions $M / C$ and $M$ are equivalent, we see by (2.3) that

$$
\|\omega \mu\|_{L_{\mu}^{M}} \leq(C+1)\|\omega u\|_{L_{\mu}^{M / C}} \leq(C+1) A=(C+1)\left\|u^{\prime}\right\|_{L_{\mu}^{M}} .
$$

Therefore the result follows.

REMARK 4.2. One can compare our results with those recently proven in [9]. Namely, in Theorem 3.1, p. 416 there, the authors obtain the following inequality for the Gaussian measure, for $M(\lambda)=\lambda^{p}$ :

$$
\int_{\mathbb{R}_{+}}|\omega u|^{p} \exp \left(-\frac{x^{2}}{2}\right) d x \leq\left(\frac{p}{p-1}\right)^{p} \int_{\mathbb{R}_{+}}\left|u^{\prime}\right|^{p} \exp \left(-\frac{x^{2}}{2}\right) d x
$$

where

$$
\omega(x)=\frac{\exp \left(x^{2} / 2(p-1)\right)}{\int_{0}^{x} \exp \left(\sigma^{2} / 2(p-1)\right) d \sigma},
$$

holding for every $u \in W_{0}^{1, p}\left(\mathbb{R}_{+}, d \mu\right.$ ) (the completion of $C_{0}^{\infty}\left(\mathbb{R}_{+}\right)$in the weighted Sobolev space $W^{1, p}\left(\mathbb{R}_{+}, d \mu\right)$, where $\mu(x)=\exp \left(-x^{2} / 2\right) d x$ is the Gaussian measure). In this case we have $\varphi(x)=x^{2} / 2$ and the quantity $\omega(x) / \varphi^{\prime}(x)$ is not bounded as required by our Theorem 3.1. Instead, the weight $\omega$ obeys a different requirement, namely the ODE $x \omega-(p-1) \omega^{\prime}=$ $(p-1) \omega^{2}$.

\section{ANALYSIS OF SETS $\mathcal{R}$}

5.1. Verification of the condition $u \in \mathcal{R}$. The purpose of this subsection is two-fold. First we give an easy practical method to verify whether $u \in \mathcal{R}$ (here $\mathcal{R}$ stands for $\mathcal{R}^{+}$or $\mathcal{R}^{-}$). Further, we discuss when the condition

$$
\int_{\mathbb{R}_{+}} M\left(\left|u^{\prime}(r)\right|\right) \exp (-\varphi(r)) d r<\infty
$$

together with $u \in \mathcal{H}$ (respectively $u \in \mathcal{H}^{*}$ ) implies that $u \in \mathcal{R}$.

We start with the following result.

Proposition 5.1. Suppose $M, \varphi$ satisfy the conditions $(\mathrm{M}),(\mu),(\omega)$, $\varphi \in C^{2}\left(\mathbb{R}_{+}\right), \varphi^{\prime}$ never vanishes and $L<\infty$ (see (2.9)). Then the following statements hold true. 
(i) Assume that $\varphi^{\prime}(R) \rightarrow 0$ as $R \rightarrow \infty$, and $u \in W_{\text {loc }}^{1,1}\left(\mathbb{R}_{+}\right)$is such that $u(R)$ and $u(R) e^{-\varphi(R)}$ are bounded for $R \rightarrow \infty$. Then for $\varphi^{\prime}<0$ we have $u \in \mathcal{R}_{(\omega, \varphi, M)}^{+}$, while for $\varphi^{\prime}>0$ we have $u \in \mathcal{R}_{(\omega, \varphi, M)}^{-}$.

(ii) Assume that $\varphi^{\prime}(r) \rightarrow 0$ as $r \rightarrow 0$, and $u \in W_{\text {loc }}^{1,1}\left(\mathbb{R}_{+}\right)$is such that $u(r)$ and $u(r) e^{-\varphi(r)}$ are bounded for $r \rightarrow 0$. Then for $\varphi^{\prime}>0$ we have $u \in \mathcal{R}_{(\omega, \varphi, M)}^{+}$, while for $\varphi^{\prime}<0$ we have $u \in \mathcal{R}_{(\omega, \varphi, M)}^{-}$.

Proof. (i) We have ( $h^{u}$ was defined in (2.10))

$$
\begin{aligned}
\left|h^{u}(R)\right| \exp (-\varphi(R)) & =\frac{M(\omega(R)|u(R)|)}{\left|\varphi^{\prime}(R)\right|} \exp (-\varphi(R)) \\
& =\frac{M\left(\left|\varphi^{\prime}(R)\right||u(R)| \frac{\omega(R)}{\left|\varphi^{\prime}(R)\right|}\right)}{\left|\varphi^{\prime}(R)\right|} \exp (-\varphi(R)) \\
& \leq c(L) \frac{M\left(\left|\varphi^{\prime}(R)\right||u(R)|\right)}{\left|\varphi^{\prime}(R)\right|} \exp (-\varphi(R)) \chi_{\{u(R) \neq 0\}}(R),
\end{aligned}
$$

where $c(\cdot)$ is defined in (4.2). The property $M(r) / r \rightarrow 0$ as $r \rightarrow 0$ and our assumptions imply

$$
\frac{M\left(\left|\varphi^{\prime}(R)\right||u(R)|\right)}{\left|\varphi^{\prime}(R)\right||u(R)|} \chi_{\{u(R) \neq 0\}}(R) \rightarrow 0 \quad \text { as } R \rightarrow \infty
$$

and so $c(L)|u(R)| \exp (-\varphi(R))$ is bounded for $R \rightarrow \infty$. Therefore $\left|h^{u}(R)\right|$ $\times \exp (-\varphi(R)) \rightarrow 0$ as $R \rightarrow \infty$ and the statement now follows from the definition of $\mathcal{R}^{+}$and $\mathcal{R}^{-}$.

(ii) As in the proof of (i), we check that $\left|h^{u}(r)\right| \exp (-\varphi(r)) \rightarrow 0$ as $r \rightarrow 0$.

In the remaining part of this subsection we examine the property (5.1). Let us set some additional notation. First, recalling $c(\cdot)$ from $(4.2)$, define, for $r>0$,

$$
\begin{aligned}
f_{\varphi}(r) & =c^{-1}\left(e^{-\varphi(r)}\right), \\
A_{\varphi}(r) & =\left\|1 / f_{\varphi}\right\|_{L^{\left(M^{*}\right)}(0, r)}, \quad B_{\varphi}(r)=\left\|1 / f_{\varphi}\right\|_{L^{\left(M^{*}\right)}(r, \infty)} .
\end{aligned}
$$

The norms here are the dual norms defined by (2.4).

We will distinguish the cases when $A_{\varphi}$ is well defined, and when $B_{\varphi}$ is well defined.

Case 1. Assume that $A_{\varphi}(r)$ is well defined for small $r$ 's. We now analyze when $u \in \mathcal{H}$ which satisfies (5.1) belongs to $\mathcal{R}$.

We start with the following lemma.

Lemma 5.1. Assume that $M, \varphi, \omega$ satisfy $(\mathrm{M}),(\varphi),(\omega)$, and the function

$$
K(r)=\frac{M\left(\omega(r) A_{\varphi}(r)\right)}{\left|\varphi^{\prime}(r)\right|} e^{-\varphi(r)}
$$


is bounded for $r \rightarrow 0$. Then for every $u \in W_{\mathrm{loc}}^{1,1}\left(\mathbb{R}_{+}\right)$such that

$$
\int_{0}^{\infty} M\left(\left|u^{\prime}(r)\right|\right) e^{-\varphi(r)} d r<\infty \quad \text { and } \quad \lim _{r \rightarrow 0} u(r)=0
$$

the function $h^{u}$ defined by (2.10) satisfies

$$
\lim _{r \rightarrow 0} h^{u}(r) e^{-\varphi(r)}=0 .
$$

Proof. Set

$$
U(r)=\int_{0}^{r}\left|u^{\prime}(\varrho)\right| d \varrho
$$

From inequality (2.5) we have

$$
\begin{aligned}
U(r) & =\int_{0}^{r}\left|u^{\prime}(\varrho)\right| d \varrho=\int_{0}^{r}\left|u^{\prime}(\varrho) f_{\varphi}(\varrho)\right| \cdot \frac{1}{\left|f_{\varphi}(\varrho)\right|} d \varrho \\
& \leq\left\|u^{\prime} \cdot f_{\varphi}\right\|_{L^{(M)}(0, r)} \cdot A_{\varphi}(r) .
\end{aligned}
$$

We have

$$
\left\|u^{\prime} f_{\varphi}\right\|_{L^{M}(0, r)} \leq\left\|u^{\prime}\right\|_{L_{\mu}^{M}(0, r)} .
$$

Indeed, if $A=\left\|u^{\prime} f_{\varphi}\right\|_{L^{M}(0, r)}$ then the definition (5.2) of $f_{\varphi}$ and the property (4.2) yield

$$
1=\int_{(0, r)} M\left(\frac{\left|u^{\prime}\right| f_{\varphi}}{A}\right) d x \leq \int_{(0, r)} c\left(f_{\varphi}\right) M\left(\frac{\left|u^{\prime}\right|}{A}\right) d x=\int_{(0, r)} M\left(\frac{\left|u^{\prime}\right|}{A}\right) \exp (-\varphi) d x .
$$

Therefore $A \leq\left\|u^{\prime}\right\|_{L_{\mu}^{M}(0, r)}$, which is (5.5).

As the dual and Luxemburg norms are equivalent, we get

$$
\left\|u^{\prime} f_{\varphi}\right\|_{L^{(M)}(0, r)} \leq A\left\|u^{\prime}\right\|_{L_{\mu}^{(M)}(0, r)} \leq A\left\|u^{\prime}\right\|_{L_{\mu}^{(M)}(0, \infty)}<\infty,
$$

where $A$ is some universal constant. Therefore $U$ is well defined.

From the assumption $\lim _{r \rightarrow 0} u(r)=0$ we get $|u(r)| \leq U(r)$, and the estimate (5.4) holds true for $u$ in place of $U$ as well.

Now,

$$
\begin{aligned}
\left|h^{u}(r) e^{-\varphi(r)}\right| & =\frac{M(\omega(r)|u(r)|)}{\left|\varphi^{\prime}(r)\right|} e^{-\varphi(r)} \leq \frac{M\left(\omega(r)\left\|u^{\prime}\right\|_{L_{\mu}^{(M)}(0, r)} A_{\varphi}(r)\right)}{\left|\varphi^{\prime}(r)\right|} e^{-\varphi(r)} \\
& \leq \frac{M\left(\omega(r) A_{\varphi}(r)\right)}{\left|\varphi^{\prime}(r)\right|} e^{-\varphi(r)} c\left(\left\|u^{\prime}\right\|_{L_{\mu}^{(M)}(0, r)}\right)=K(r) c\left(\left\|u^{\prime}\right\|_{L_{\mu}^{(M)}(0, r)}\right) .
\end{aligned}
$$

But since $c(x) \rightarrow 0$ as $x \rightarrow 0$, and $\left\|u^{\prime}\right\|_{L_{\mu}^{(M)}(0, r)} \rightarrow 0$ as $r \rightarrow 0$, the assertion (5.3) follows from the boundedness of $K(r)$ for small $r$ 's.

As a corollary, we obtain straight from the definition of $\mathcal{R}^{+}, \mathcal{R}^{-}$: 
COROLlary 5.1. Suppose that the assumptions of Lemma 5.1 are satisfied.

(i) If $\varphi^{\prime}>0$, then

$$
\left\{u \in \mathcal{H}: \int_{\mathbb{R}_{+}} M\left(\left|u^{\prime}(r)\right|\right) \exp (-\varphi(r)) d r<\infty\right\} \subset \mathcal{R}^{+} .
$$

(ii) If $\varphi^{\prime}<0$, then

$$
\left\{u \in \mathcal{H}: \int_{\mathbb{R}_{+}} M\left(\left|u^{\prime}(r)\right|\right) \exp (-\varphi(r)) d r<\infty\right\} \subset \mathcal{R}^{-} .
$$

To illustrate the statements above we now discuss the following example.

EXAmple 5.1. Let $\varphi(r)=-\alpha \ln r, \alpha<p-1, M(\lambda)=\lambda^{p}$ and $\omega(r)=1 / r$ as in Theorem 3.1. Then $M^{*}(\lambda)=c_{p} \lambda^{p /(p-1)}$ and, in this range of $\alpha$ 's,

$$
f_{\varphi}(r)=r^{\alpha / p}, \quad M^{*}\left(f_{\varphi}(r)\right)=c_{p} r^{\alpha /(p-1)}, \quad A_{\varphi}(r)=a_{p} r^{(p-1-\alpha) / p} .
$$

Thus $K(r)$ is just a constant. Moreover, every $u \in \mathcal{H}$ with $\int_{0}^{\infty}\left|u^{\prime}\right|^{p} x^{\alpha} d x<\infty$ belongs to $\mathcal{R}_{\left(1 / x, x^{\alpha}, \lambda^{p}\right)}^{-}$for $\alpha<0$, and to $\mathcal{R}_{\left(1 / x, x^{\alpha}, \lambda^{p}\right)}^{+}$when $\alpha>0$.

CASE 2. We now assume that $B_{\varphi}(R)$ is well defined for large $R$ 's. In this case we have the following dual statements dealing with the question whether $u \in \mathcal{H}^{*}$ which satisfies (5.1) belongs to $\mathcal{R}$.

Lemma 5.2. Assume that $M, \varphi, \omega$ satisfy $(\mathrm{M}),(\varphi),(\omega)$, and the function

$$
L(R)=\frac{M\left(\omega(R) B_{\varphi}(R)\right)}{\left|\varphi^{\prime}(R)\right|} e^{-\varphi(R)}
$$

is bounded for $R \rightarrow \infty$. Then for every $u \in \mathcal{H}^{*}$ with $\int_{0}^{\infty} M\left(\left|u^{\prime}(r)\right|\right) e^{-\varphi(r)} d r$ $<\infty$ the function $h^{u}$ defined by (2.10) satisfies

$$
\lim _{R \rightarrow \infty} h^{u}(R) e^{-\varphi(R)}=0 .
$$

Proof. It is almost identical to that of Lemma 5.1: just replace $U(r)=$ $\int_{0}^{r}\left|u^{\prime}(\varrho)\right| d \varrho$ with $U^{*}(R)=\int_{R}^{\infty}\left|u^{\prime}(\varrho)\right| d \varrho$ and proceed as before.

As a counterpart of Corollary 5.1 we have the following.

Corollary 5.2. Suppose that the assumptions of Lemma 5.2 are satisfied.

(i) If $\varphi^{\prime}>0$, then

$$
\left\{u \in \mathcal{H}^{*}: \int_{\mathbb{R}_{+}} M\left(\left|u^{\prime}(r)\right|\right) \exp (-\varphi(r)) d r<\infty\right\} \subset \mathcal{R}^{-} .
$$

(ii) If $\varphi^{\prime}<0$, then

$$
\left\{u \in \mathcal{H}^{*}: \int_{\mathbb{R}_{+}} M\left(\left|u^{\prime}(r)\right|\right) \exp (-\varphi(r)) d r<\infty\right\} \subset \mathcal{R}^{+} .
$$


This result is illustrated by the following example.

EXAMPLE 5.2. We again consider the case $\varphi(r)=-\alpha \ln r, M(\lambda)=\lambda^{p}$ and $\omega(r)=1 / r$ as in Theorem 3.1, but now with $\alpha>p-1$. We have $B_{\varphi}(r)=b_{p} r^{(-\alpha+p-1) / p}$ and $L(r)$ is a constant. Therefore every $u \in \mathcal{H}^{*}$ which satisfies $\int_{0}^{\infty}\left|u^{\prime}\right|^{p} x^{\alpha} d x<\infty$ belongs to $\mathcal{R}_{\left(1 / x, x^{\alpha}, \lambda^{p}\right)}^{+}$.

REMARK 5.1. Suppose that the assumptions of Theorem 2.1 are satisfied and put $\mathcal{R}=\mathcal{R}^{+}$in the case of (B1) and $\mathcal{R}=\mathcal{R}^{-}$in the case of (B2). One could ask whether the spaces

$$
\left\{u \in \mathcal{R}: \int_{\mathbb{R}_{+}} M\left(\left|u^{\prime}(r)\right|\right) \exp (-\varphi(r)) d r<\infty\right\},
$$

playing the crucial role in the inequality (2.13), can possibly be nonlinear. We do not know the answer to this question.

5.2. Relation to Bloom-Kerman results. We now compare our results with those of Bloom and Kerman [6].

5.2.1. When our conditions imply Bloom and Kerman's. Here we give an example where our assumptions yield inequality (3.5) for all functions $T f=\int_{0}^{t} f(\tau) d \tau \in \mathcal{H}$, so the Bloom-Kerman condition is satisfied.

Corollary 5.1 yields the following proposition.

Proposition 5.2. Assume that $(M, \varphi, \omega)$ satisfy the assumptions of Lemma 5.1 and either $\left(\varphi^{\prime}>0, b_{1}>0, L<\infty\right)$ or $\left(\varphi^{\prime}<0, b_{2}>0, L<\infty\right)$. Then:

(i) There exists a constant $C>0$ such that

$$
\begin{aligned}
& \int_{0}^{\infty} M(\omega(x)|u(x)|) \exp (-\varphi(x)) d x \leq C \int_{0}^{\infty} M\left(\left|u^{\prime}(x)\right|\right) \exp (-\varphi(x)) d x \\
& \text { for every } u \in \mathcal{H} .
\end{aligned}
$$

(ii) The triple $(\omega, \varphi, M)$ satisfies the Bloom-Kerman condition:

$$
\int_{0}^{y} M^{*}\left(\frac{G(\varepsilon, y)}{B \varepsilon \exp (-\varphi(x))}\right) \exp (-\varphi(x)) d x \leq G(\varepsilon, y)<\infty,
$$

for all $y>0$ and $\varepsilon>0$, where

$$
G(\varepsilon, y)=\int_{y}^{\infty} M(\varepsilon \omega(x)) \exp (-\varphi(x)) d x
$$

and $B>0$ is a constant. 
Proof. (i) This is just a combination of Corollary 5.1 and Theorem 2.1.

(ii) Let $u(x)=\int_{0}^{x} f(\tau) d \tau=(T f)(x) \in \mathcal{H}$ be the Hardy transform of $f$. Then (i) implies

$$
\int_{0}^{\infty} M(\omega(x) T f(x)) \exp (-\varphi(x)) d x \leq C \int_{0}^{\infty} M(f(x)) \exp (-\varphi(x)) d x,
$$

which is equivalent to (5.6) (see Theorem 1.7 in [6] and our comments in Subsection 3.2).

5.2.2. When Bloom and Kerman conditions are not satisfied. It may happen that our conditions are satisfied and the Bloom-Kerman conditions are not. In that case inequalities (2.13) cannot hold for every Hardy transform $u \in \mathcal{H}$ (see (1.3)) but they hold on proper subsets in the set of Hardy transforms. This is illustrated by the following result.

Proposition 5.3. There exists a triple $(\omega, \varphi, M)$ such that conditions $(\mathrm{M}),(\mu),(\omega)$ are satisfied and:

(i) $(\omega, \varphi, M)$ satisfies $(\mathrm{B} 1)$, in particular

$$
\left.\int_{\mathbb{R}_{+}} M(\omega(r))|u(r)|\right) \mu(d r) \leq C \int_{\mathbb{R}_{+}} M\left(\left|u^{\prime}(r)\right|\right) \mu(d r)
$$

for all $u \in \mathcal{R}_{(\omega, \varphi, M)}^{+}$.

(ii) $(\omega, \varphi, M)$ does not satisfy the Bloom-Kerman condition (5.6).

(iii) The set

$$
\mathcal{R}_{(\omega, \varphi, M)}^{(0,+)}:=\mathcal{R}_{(\omega, \varphi, M)}^{+} \cap \mathcal{H} \subseteq \mathcal{H}
$$

is a proper subset of $\mathcal{H}$. Moreover, there is no constant $C$ such that inequality (5.7) is satisfied for every $u \in \mathcal{H}$.

Proof. Let $p>1$ and

$$
M(\lambda)=\lambda^{p}, \quad \varphi(x)=-\frac{1}{2} x^{2}, \quad \omega(x)=x .
$$

In particular, conditions $(\mathrm{M}),(\mu),(\omega)$ are satisfied, $b_{1}=1+\frac{1}{x^{2}}(p-1)>0$, $L=1$ and condition (B1) is also satisfied. Therefore (i) follows by Theorem 2.1 .

(ii) The Bloom-Kerman condition does not hold: one has

$$
G(\varepsilon, y)=\int_{y}^{\infty}(\varepsilon x)^{p} e^{x^{2} / 2} d x=\infty
$$

and so (5.6) is violated.

(iii) The Laplace function

$$
u(r)=\int_{0}^{r} \exp \left(-\tau^{2}\right) d \tau
$$

belongs to $\mathcal{H} \backslash \mathcal{R}_{(\omega, \varphi, M)}^{0,+}$ and does not satisfy (5.7). 
Acknowledgements. The authors would like to thank Miroslav Krbec for helpful advice and discussions.

The work of both authors was supported by a KBN grant no. 1-PO3A008-29. It was also partially supported by EC FP6 Marie Curie programmes SPADE2 and CODY.

\section{References}

[1] H. D. Alber, Materials with Memory. Initial-Boundary Value Problems for Constitutive Equations with Internal Variables, Lecture Notes in Math. 1682, Springer, 1998.

[2] A. Alvino, V. Ferone and G. Trombetti, On the properties of some nonlinear eigenvalues, SIAM J. Math. Anal. 29 (1998), 437-451.

[3] J. M. Ball, Constitutive inequalities and existence theorems in nonlinear elastostatics, in: Nonlinear Analysis and Mechanics: Heriot-Watt Symposium (Edinburgh, 1976), Vol. I, Res. Notes Math. 17, Pitman, London, 1977, 187-241.

[4] P. R. Beesack, Integral inequalities of the Wirtinger type, Duke Math. J. 25 (1958), 477-498.

[5] S. Bloom and R. Kerman, Weighted norm inequalities for operators of Hardy type, Proc. Amer. Math. Soc. 113 (1991), 135-141.

[6] - , - Weighted Orlicz space integral inequalities for the Hardy-Littlewood maximal operator, Studia Math. 110 (1994), 149-167.

[7] D. W. Boyd, Indices for the Orlicz spaces, Pacific J. Math. 38 (1971), 315-323.

[8] J. S. Bradley, Hardy's inequalities with mixed norms, Canad. Math. Bull. 21 (1978), 405-408.

[9] B. Brandolini, F. Chiacchio and C. Trombetti, Hardy type inequalities and Gaussian measure, Comm. Pure Appl. Anal. 6 (2007), 411-428.

[10] H. Brezis and J. L. Vázquez, Blow-up solutions of some nonlinear elliptic problems, Rev. Mat. Univ. Complut. Madrid 10 (1997), 443-469.

[11] X. Cabré and Y. Martel, Weak eigenfunctions for the linearization of extremal elliptic problems, J. Funct. Anal. 156 (1998), 30-56.

[12] R. Caccioppoli, Limitazioni integrali per le soluzioni di un'equazione lineare ellitica a derivate parziali, Giorn. Mat. Battaglini (4) 4 (80) (1951), 186-212.

[13] J. Chabrowski and M. Willem, Hardy's inequality on exterior domains, Proc. Amer. Math. Soc. 134 (2006), 1019-1022.

[14] K. Chełmiński, On monotone plastic constitutive equations with polynomial growth condition, Math. Methods Appl. Sci. 22 (1999), 547-562.

[15] A. Cianchi, Some results in the theory of Orlicz spaces and applications to variational problems, in: Nonlinear Analysis, Function Spaces and Applications, Vol. 6 (Prague, 1998), Acad. Sci. Czech Rep., Prague, 1999, 50-92.

[16] W. J. Coles, A general Wirtinger-type inequality, Duke Math. J. 27 (1960), 133-138.

[17] B. Dyda, A fractional order Hardy inequality, Illinois J. Math. 48 (2004), 575-588.

[18] A. Fiorenza and M. Krbec, Indices of Orlicz spaces and some applications, Comment. Math. Univ. Carolin. 38 (1997), 433-451. 
[19] P. J. Fitzsimmons, Hardy's inequality for Dirichlet forms, J. Math. Anal. Appl. 250 (2000), 548-560.

[20] B. Florkiewicz, Some integral inequalities of Hardy type, Colloq. Math. 43 (1980), 321-330.

[21] B. Florkiewicz and K. Wojteczek, Some second-order integral inequalities of generalized Hardy type, Proc. Roy. Soc. Edinburgh Sect. A 129 (1999), 947-958.

[22] J. P. García Azorero and A. I. Peral, Hardy inequalities and some critical elliptic and parabolic problems, J. Differential Equations 144 (1998), 441-476.

[23] A. Gogatishvili, B. Opic and L. Pick, Weighted inequalities for Hardy-type operators involving suprema, Collect. Math. 57 (2006), 227-255.

[24] J. Gustavsson and J. Peetre, Interpolation of Orlicz spaces, Studia Math. 60 (1977), $33-59$.

[25] G. H. Hardy, Note on a theorem of Hilbert, Math. Z. 6 (1920), 314-317.

[26] G. H. Hardy, J. E. Littlewood and G. Pólya, Inequalities, Cambridge Univ. Press, 1952 .

[27] H. P. Heinig and Q. S. Lai, Weighted modular inequalities for Hardy-type operators on monotone functions, J. Inequal. Pure Appl. Math. 1 (2000), no. 1, art. 10, 25 pp.

[28] H. Heinig and L. Maligranda, Interpolation with weights in Orlicz spaces, Boll. Un. Mat. Ital. B (7) 8 (1994), 37-55.

[29] T. Iwaniec and C. Sbordone, Caccioppoli estimates and very weak solutions of elliptic equations, in: Renato Caccioppoli and Modern Analysis, Atti Accad. Naz. Lincei Cl. Sci. Fis. Mat. Natur. Rend. Lincei (9) Mat. Appl. 14 (2003), 189-205.

[30] A. Kałamajska and K. Pietruska-Pałuba, Gagliardo-Nirenberg inequalities in weighted Orlicz spaces, Studia Math. 173 (2006), 49-71.

[31] S. S. Kazarian, Integral inequalities in Orlicz reflexive weighted spaces for the conjugate function, Dokl. Akad. Nauk Armyan. SSR 25 (1990), 261-273 (in Russian).

[32] R. Kerman and A. Torchinsky, Integral inequalities with weights for the Hardy maximal function, Studia Math. 71 (1981/82), 277-284.

[33] V. Kokilashvili, On Hardy's inequalities in weighted spaces, Soobshch. Akad. Nauk Gruzin. SSR 96 (1979), 37-40 (in Russian).

[34] V. Kokilashvili and M. Krbec, Weighted Inequalities in Lorentz and Orlicz Spaces, World Sci., Singapore, 1991.

[35] M. A. Krasnosel'skiı̌ and Ya. B. Rutickiı̌, Convex Functions and Orlicz Spaces, Noordhoff, Groningen, 1961.

[36] A. Kufner, Weighted Sobolev Spaces, Wiley, Chichester, 1985.

[37] A. Kufner and B. Opic, Hardy-type Inequalities, Longman Sci. Tech., Harlow, 1990.

[38] A. Kufner and L. E. Persson, Weighted Inequalities of Hardy Type, World Sci., River Edge, NJ, 2003.

[39] A. Kufner and A. M. Sändig, Some Applications of Weighted Sobolev Spaces, Teubner-Texte Math. 100, Treubner, Leipzig, 1987.

[40] Q. S. Lai, Two weight mixed $\Phi$-inequalities for the Hardy operator and the HardyLittlewood maximal operator, J. London Math. Soc. 48 (1992), 301-318.

[41] - Two weight $\Phi$-inequalities for the Hardy operator, the Hardy-Littlewood maximal operator and fractional integrals, Proc. Amer. Math. Soc. 118 (1993), 129-142.

[42] - Weighted integral inequalities for the Hardy type operator and the fractional maximal operator, J. London Math. Soc. 49 (1994), 244-266. 
[43] Q. S. Lai, Weighted modular inequalities for Hardy type operators, Proc. London Math. Soc. 79 (1999), 649-672.

[44] V. G. Maz'ya, Sobolev Spaces, Springer, 1985.

[45] D. S. Mitrinović, J. E. Pečarić and A. M. Fink, Inequalities Involving Functions and Their Derivatives, Kluwer, Dordrecht, 1991.

[46] B. Muckenhoupt, Hardy's inequality with weights, Studia Math. 44 (1972), 31-38.

[47] C. A. Okpoti, L. E. Persson and G. Sinnamon, An equivalence theorem for some integral conditions with general measures related to Hardy's inequality, J. Math. Anal. 326 (2007), 398-413.

[48] W. Pompe, Quasistatic and dynamic problems in viscoplasticity theory - nonlinearities with power growth, Math. Methods Appl. Sci. 27 (2004), 1347-1365.

[49] M. M. Rao and Z. D. Ren, Theory of Orlicz Spaces, Dekker, New York, 1991.

[50] M. Rao and H. Šikić, Potential-theoretic nature of Hardy's inequality for Dirichlet forms, J. Math. Anal. Appl. 318 (2006), 781-786.

[51] E. T. Sawyer, Weigted Lebesgue and Lorentz norm inequalities for the Hardy operator, Trans. Amer. Math. Soc. 281 (1984), 329-337.

[52] I. B. Simonenko, Interpolation and extrapolation of linear operators in Orlicz spaces, Mat. Sb. (N.S.) 63 (105) (1964), 536-553 (in Russian).

[53] G. Sinnamon, Weighted Hardy and Opial-type inequalities, J. Math. Anal. Appl. 160 (1991), 434-445.

[54] V. D. Stepanov, Weighted inequalities for a class of Volterra convolution operators, J. London Math. Soc. 45 (1992), 232-242.

[55] E. W. Stredulinsky, Weighted Norm Inequalities and Degenerate Elliptic Partial Differential Equations, Lecture Notes in Math. 1074, Springer, Berlin, 1984.

[56] L. Tartar, Imbedding theorems of Sobolev spaces into Lorentz spaces, Boll. Un. Mat. Ital. Sez. B (8) 1 (1998), 479-500.

[57] J. L. Vázquez and E. Zuazua, The Hardy inequality and the asymptotic behaviour of the heat equation with an inverse-square potential, J. Funct. Anal. 173 (2000), $103-153$.

[58] Y. Q. Yan, Relationship between Matuszewska-Orlicz, Semenov and Simonenko indices of $\varphi$-functions, Publ. Inst. Math. (Beograd) (N.S.) 73 (87) (2003), 139-147.

Institute of Mathematics

University of Warsaw

Banacha 2

02-097 Warszawa, Poland

E-mail: kalamajs@mimuw.edu.pl

kpp@mimuw.edu.pl

Received June 26, 2007

Revised version March 11, 2009 